Article

\title{
Joint Trajectory and Scheduling Optimization for The Mobile UAV Aerial Base Station: A Fairness Version
}

\author{
Yancheng Chen, Ning Li *, Xijian Zhong and Wei Xie \\ College of Communications Engineering, PLA Army Engineering University, Nanjing 210007, China \\ * Correspondence: js_ningli@163.com; Tel.: +86-173-2708-7039
}

Received: 13 June 2019; Accepted: 29 July 2019; Published: 31 July 2019

check for updates

\begin{abstract}
Recently, unmanned aerial vehicles (UAVs) have been widely studied in the communication area to work as aerial base stations, due to the high probability of line of sight (LoS) and high flexibility. However, few works consider fairness for the users, which is one of the most important metrics for a network. In this paper, in order to maximize network capacity with the consideration of fairness, trajectory and scheduling of the mobile UAV aerial base station are jointly optimized. Firstly, the problem of maximizing network capacity with the consideration of fairness is formulated. On account of the coupling relationship of trajectory and scheduling, an alternate iteration approach that contains ant colony algorithm and genetic algorithm are then proposed to solve this intractable problem. Finally, the simulation results demonstrate the fairness enhance of the network and the validity and effectiveness of the proposed optimization approach.
\end{abstract}

Keywords: unmanned aerial vehicle (UAV); fairness performance; circular trajectory; optimization; ant colony algorithm; genetic algorithm

\section{Introduction}

Recently, unmanned aerial vehicles (UAVs) have attracted wide attention in many research fields for their ability to provide wireless service [1]. Using UAVs as base stations is considered a promising way to improve wireless network coverage and rate performance. Moreover, UAV-BSs can assist in terrestrial communication in a variety of scenarios, such as surveillance, monitoring, aerial imaging, and cargo delivery. UAVs can be deployed to assist the existing communication infrastructure when ground stations are unable to provide communication services, due to various incidents [2]. For example, the base station cannot work normally, due to a fault or the network congestion caused by high user density. UAVs cannot only be used as wireless relay to improve a wireless connection and cover the ground device, but also as an aerial base station to provide reliable downlink and uplink communication for ground users [3].

At present, the research of mobile UAV as an aerial base station is increasing day by day. In Reference [4], the coexistence of UAVs and D2D networks transmitting data in downlink is studied. The work of Reference [5] shows that for cases of Rician fading, there exists optimum height that maximizes the coverage area. In References [6,7], the coverage probability performance of multiple UAVs is studied, and the flexibility of the scalable dynamic network is considered. In Reference [8], a new method is proposed to deploy UAVs to collect uplink data from mobile ground IoT devices. The work in [9] considers the deployment of two UAVs with and without interference. The work in Reference [10] studied how to reduce the transmission energy of UAVs, while meeting the requirement of users. In Reference [11], the trajectory and heading of multiple UAVs are deduced when the ground user is stationary. The work in Reference [12] improves the network throughput significantly by 
jointly optimizing relay trajectory and power distribution. The work in Reference [13] studies the 3D deployment of multiple UAVs in consideration of fairness. The work in Reference [14] established the channel model of LoS probability, and the relation between maximum coverage radius and height is given. The work in Reference [15] studied the deployment of the UAV in a novel way. The work in Reference [16] studied the emergency deployment of UAV base station to minimize transmission delays. The work in Reference [17] studied the deployment of UAVs in both horizontal and vertical dimensions. The work in Reference [18] studies the 3D deployment of the UAV in the case that users have different QoS. The work in Reference [19] maximized the weighted sum-rate for the downlink transmission in Multi-cell Multi-association OFDMA heterogeneous networks. The work of Reference [20] studies UAV's energy-saving deployment can optimize the trajectory of UAV. The work of Reference [21] proposed a new cyclical multiple access scheme.

UAVs are able to change its location based on user requirements, which is quite different from ground base stations. Although UAV-BS haves many advantages, many challenges remain in UAV-aided communication. Fairness is an important indicator metrics in communication or disaster-affected network, and they should be considered for the trajectory of the UAV-BS [13]. According to the characteristics of the air-to-ground channel model, users far away from UAV-BS have higher path losses than users close to the UAV-BS [14]. Therefore, the QoS requirements of users at the edge of the coverage area cannot be reached; that is, the UAV-BS cannot provide wireless service for users at the edge of the coverage area. The deployment of multiple UAVs with the consideration of fairness is studied in Reference [13]. However, multiple UAVs are needed, and the hardware costs are high. The total logarithm rate of users is considered in Reference [13], and the fairness of the user transmission rate is measured by optimizing the average logarithm rate of users connected to each UAV. We consider a single UAV to serve ground users taking advantage of the mobility of the UAV. In order to maximize network capacity with the consideration of fairness, trajectory and scheduling of the mobile UAV aerial base station are jointly optimized.

In this paper, we propose a novel approach for optimizing the trajectory and scheduling of the UAV to provide wireless to service ground users while maximizing network capacity with the consideration of fairness. We consider the business requirements for delay tolerance, so the number of slots divided over a hovering period is equal to the number of users. So that all users in the area can be served, we considered a UAV-BS hovering with a circular trajectory. We divided the period of the UAV-BS into time slots according to the number of users, and each user only uses one slot. We take the square of the difference between the average signal-to-noise ratio of each user within a time slot and the maximal signal-to-noise ratio available to the user as the metric to measure fairness. Our goal is to maximize fairness by jointly optimizing the hovering radius of the UAV-BS and the users schedule. The formulated problem is a mixed-integer non-convex optimization problem that is challenging to solve. In addition, we adopted the ant colony algorithm and the genetic algorithm to optimize the above two parameters.

The main contribution of this paper as follows: Firstly, we considered a UAV-BS hovering with a circular trajectory so that all users in the area can be served. Joint trajectory and scheduling optimization are considered to maximize the network capacity with the consideration of fairness. Secondly, an alternate iteration approach that contains ant colony algorithm and genetic algorithm are proposed to solve this problem. Finally, the simulation results evaluate our algorithm.

\section{System Model}

To set up the system model, a large area containing the number of users can be considered. The terrestrial base stations are failed to work, due to some accident. In order to solve the communication problems of ground users, a UAV-BS is hovered over the target area to provide wireless service. The hovers in a circular trajectory with a radius of $r$ and the period are $T$. Let $u$ represent the set of users that UAV-BS needs to serve. Let $\left(x_{i}, y_{i}\right)$ denote the horizontal location of the users $i$; and the UAV locate at $\left(x_{0}, y_{0}, h\right)$. 
The air-to-ground (A2G) channel model proposed by the author's name [4] is adopted. The path losses of Line-of-Sight (LoS) $L_{L o S}$ and non-Line-of-Sight (NLoS) $L_{N L o S}$ are respectively proposed in Reference [4]:

$$
\begin{aligned}
L_{\text {LoS }} & =20 \log \left(\frac{4 \pi f d_{i}}{c}\right)+\eta_{\text {LoS }}, \\
L_{N L O S} & =20 \log \left(\frac{4 \pi f d_{i}}{c}\right)+\eta_{\text {NLoS }},
\end{aligned}
$$

where $f$ and $c$ are respectively the carrier frequency and the speed of light. The distance between the UAV-BS and user $i$ is denoted by $d_{i}=\sqrt{h^{2}+r_{i}^{2}}$, where $r_{i}=\sqrt{\left(x_{i}-x_{0}\right)^{2}+\left(y_{i}-y_{0}\right)^{2}}$. Furthermore, $\eta_{L O S}$ and $\eta_{N L O S}$ are respectively additional loss for Line-of-Sight (LoS) and non-Line-of-Sight (NLoS). The value of $\eta_{L o S}$ and $\eta_{N L O S}$ depend on the environment, and are given by Reference [4] in different environments. In addition, the Line-of-Sight (LoS) probability as follows:

$$
P_{\text {LoS }}=\frac{1}{1+\alpha \exp \left(-\beta\left(\frac{180}{\pi} \theta_{i}-\alpha\right)\right)}
$$

where $\alpha$ and $\beta$ are environmental constants, and $\theta_{i}=\arctan \left(\frac{h}{r_{i}}\right)$ is the elevation angle of the user $i$ in the set $u$. In addition, the probability of non-Line-of-Sight (NLoS) is $P_{N L o S}=1-P_{L o S}$. Let $A=\eta_{L o S}-\eta_{N L o S}$, $B=20 \log \left(\frac{4 \pi f}{c}\right)+\eta_{N L o S}$, and the probabilistic mean path loss as follows:

$$
\begin{gathered}
L=P_{L o S} L_{L o S}+P_{N L o S} L_{N L o S}, \\
L\left(h, r_{i}\right)=\frac{A}{1+\alpha \exp \left(-\beta\left(\frac{180}{\pi} \arctan \left(\frac{h}{r_{i}}\right)-\alpha\right)\right)}+10 \log \left(h^{2}+r_{i}^{2}\right)+B .
\end{gathered}
$$

Equation (5) can be further rewritten as follows:

$$
L\left(\theta_{i}, r_{i}\right)=\frac{A}{1+\alpha \exp \left(-\beta\left(\frac{180}{\pi} \theta_{i}-\alpha\right)\right)}+20 \log \left(\frac{r_{i}}{\cos \theta_{i}}\right)+B .
$$

For a given transmission power $p_{t}$, the received power $p_{r}$ at the user $i$ is: $p_{r}=p_{t}-L\left(h, r_{i}\right)$. It is assumed that the user $i$ is covered if the received power $p_{r}$ exceeds a certain threshold $p_{\min }$. If the link experiences a path loss less than a certain threshold $L_{t h}$, the user $i$ is covered by the UAV-BS. According to References $[17,18]$, for any QoS requirement $L_{t h}$, the optimal elevation angle $\theta^{*}$ that maximizes the coverage radius is a constant and only depends on the environment. In addition, $\theta^{*}=20.34^{\circ}, 42.44^{\circ}, 54.62^{\circ}, 75.52^{\circ}$ can be obtained for the suburban, urban, dense urban, and high-rise urban environments by solving Equation (6). The maximum coverage radius can also be evaluated by solving Equation (6). Finally, the optimal height $h^{*}$ of the UAV-BS can be evaluated.

Next, a scenario is discussed such that the UAV-BS at a height of $h^{*}$ hovers in a circular trajectory with a radius of $r$ and the period is $T$. In addition, the instantaneous location of the UAV-BS is assumed to be $(x, y)$. The system model is shown in Figure 1 .

Therefore, the trajectory of the UAV-BS is $x^{2}+y^{2}=r^{2}$. It can be written as a parametric equation, as follows:

$$
\begin{aligned}
& x=r \cos \theta \\
& y=r \sin \theta
\end{aligned},
$$

Because of the circular trajectory of the UAV-BS, the parameter $\theta$ is given by: $\theta(t)=\frac{2 \pi}{T} t$, and (7) can be further written as:

$$
\begin{aligned}
& x(t)=r \cos \left(\frac{2 \pi}{T} t\right) \\
& y(t)=r \cos \left(\frac{2 \pi}{T} t\right)
\end{aligned} .
$$




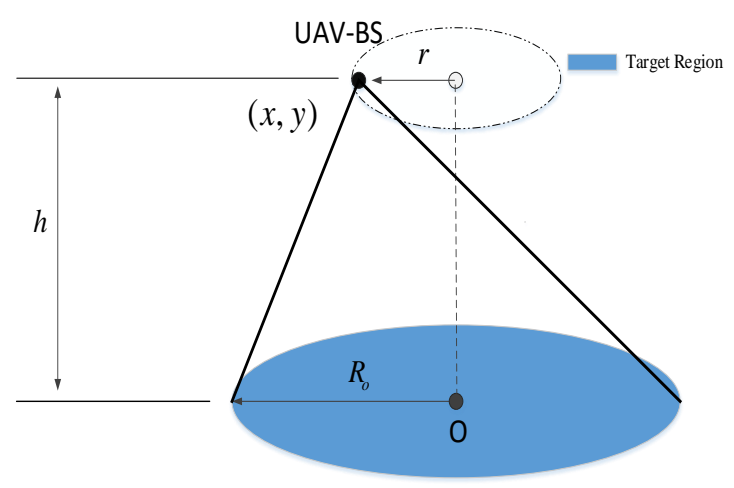

Figure 1. System model.

The received power is a function of time and can be written as:

$$
p_{r, i}(t)=p_{t}-L\left(h, r_{i}(t)\right),
$$

where $r_{i}(t)=\sqrt{\left(x_{i}-x(t)\right)^{2}+\left(y_{i}-y(t)\right)^{2}}$. In addition, the Signal-to-Noise Ratio (SNR) of a user $i$ denoted by $S N R_{i}(t)$ is:

$$
\operatorname{SNR}_{i}(t)=p_{t}-L\left(h, r_{i}(t)\right)-p_{N}
$$

where $p_{N}$ is the noise power.

The UAV-BS can only partially cover the target area at each moment, due to the large target area. To enable all users on the target area to be served in one period, the period $T$ is divided into $N$ segments according to the number of terrestrial users, where $N$ is the number of terrestrial users. The length of each time slot is $\frac{T}{N}$. A binary variable $u_{i}[n]$ is also defined, which indicates that the user $i$ is served by the UAV in the time segment $n$ if $u_{i}[n]=1$; otherwise, $u_{i}[n]=0$. As such, $u_{i}[n]$ indicates each user's connection with the UAV in each time segments. It is assumed that the UAV only serves one user in each time segment, which yields the following constraints,

$$
\begin{aligned}
& \sum_{i=1}^{N} u_{i}[n]=1, \quad \forall n \\
& \sum_{n=1}^{N} u_{i}[n]=1, \quad \forall i \\
& u_{i}[n] \in\{0,1\}, \quad \forall i, n
\end{aligned}
$$

The minimum path loss $L_{\min }$ is the path loss when the user is directly below the UAV and can be calculated, that is, $L_{\min }=\left.L\left(h, r_{i}\right)\right|_{r_{i}=0}$. The maximum SNR, can be expressed as:

$$
S N R_{\max }=p_{t}-L_{\min }-p_{N} .
$$

Furthermore, the average SNR of the user $i$ in time segment $n$ denoted by $S N R_{i}[n]$ is:

$$
\operatorname{SNR}_{i}[n]=\frac{\int_{\frac{T}{N}(n-1)}^{\frac{T}{N} n} S N R_{i}(t) d t}{T / N} .
$$

Since $S N R_{\max }$ is a fixed value, we take square of the difference between the average signal-to-noise ratio of each user within a time slot (i.e., $S N R_{i}[n]$ ) and this fixed value as the metric to measure fairness. It can be written as $\left(S N R_{\max }-S N R_{i}[n]\right)^{2}$. After UAV hovers over the target area for a period, all users obtain service in turn. The fairness metric can be further written as $\sum_{i=1}^{N} \sum_{n=1}^{N} u_{i}[n]\left(S N R_{\max }-S N R_{i}[n]\right)^{2}$. 
After UAV hovers over the target area for a period, all users obtain service in turn. Since UAV cannot completely cover users in a time slot, it is necessary to optimize the hovering radius and user scheduling to ensure the quality of service to each user. Thus, we take the square of the difference between SNR of the user and maximal SNR as the fairness metric, and can make SNR of the user as close to maximal SNR as possible. This metric indicates the degree of difference in the signal-to-noise ratio of users and smaller value means better fair performance.

Let $A=\left\{u_{i}[n], \forall i, n\right\}$. The goal is to minimize the sum of squares of the difference between average SNR $S N R_{i}[n]$ and the maximum SNR $S N R_{\max }$ by jointly optimizing user scheduling and the hovering radius of the UAV. The optimization problem can be formulated as follow:

$$
\begin{aligned}
& \min _{r, A} \sum_{i=1}^{N} \sum_{n=1}^{N} u_{i}[n]\left(S N R_{\max }-S N R_{i}[n]\right)^{2} \\
& \text { s.t } \\
& \text { a) } \sum_{i=1}^{N} u_{i}[n]=1, \quad \forall n \\
& \text { b) } \sum_{n=1}^{N} u_{i}[n]=1, \quad \forall i \\
& \text { c) } u_{i}[n] \in\{0,1\}, \quad \forall i, n \\
& \text { d) } r \leq R_{O}
\end{aligned}
$$

Problem (14) is very difficult to solve mainly for the following reasons: Firstly, the optimization variables $A$ for user scheduling are binary, and thus $(14 a-c)$ are integer constraints. Secondly, since the variables contain 0 and 1, problem (14) is non-convex optimization problem [22].

\section{Proposed Algorithm}

Problem (14) is a mixed-integer non-convex problem, which is difficult in general to be optimally solved. In order to be able to ignore the complex process and obtain the results quickly and accurately, we use a heuristic algorithm to solve Problem (14). First, the Ant Colony Algorithm (ACA) is adopted to optimize user scheduling $A$. Then, the Genetic Algorithm (GA) is used to optimize the hovering radius of the UAV.

\subsection{User Scheduling Optimization}

The ACA is a simulated evolutionary algorithm, which is a heuristic algorithm to solve combinatorial optimization. It is inspired by the foraging behavior of ants, and its inspiration comes from the behavior of ants in finding paths in the process of searching for food. The ACA has been widely applied in various fields, and it has solved many problems in combinatorial optimization, due to its characteristics of partial parallel computation and heuristic searching.

The basic idea of the ACA is as follows: The feasible solution of the problem to be optimized is represented by the ant's walking path, and the solution space of the problem to be optimized is constituted by all the paths of the entire ant colony. Ants with shorter paths release more pheromones. The concentration of pheromones accumulated on shorter paths gradually increases as the number of iterations increases, and more and more ants choose this path. Finally, the whole ant colony focuses on the optimal path under the action of positive feedback, and this solution is the optimal solution of the problem to be optimized.

For a given hovering radius $r$ of the UAV, the optimal user scheduling $A$ need to be found. When the ACA is used to solve this problem, the user is equivalent to the city in the Traveling Salesman Problem (TSP), and the value of the objective function is equivalent to the distance in TSP. Our goal is to determine the ordering of all users, that is, the optimal path of the ant, such that the sum of the objective functions is minimized.

The most classical problem solved by the ACA is the TSP. Calculating the distance between cities is a very important link when using ant colony algorithm to solve TSP, because the distance between 
cities directly affects the updating of the pheromone, thus affecting path choice of subsequent ants. In the traditional TSP, the distance between cities (also known as the value of the objective function) can be calculated before the iteration, and it will not change with the increase of the number of iterations of the algorithm. Different from traditional TSP, when solving Problem (14) with the ACA, the distance between cities with the number of iterations. Therefore, we improved the ACA to solve the problem. The algorithm process is shown in Algorithm 1.

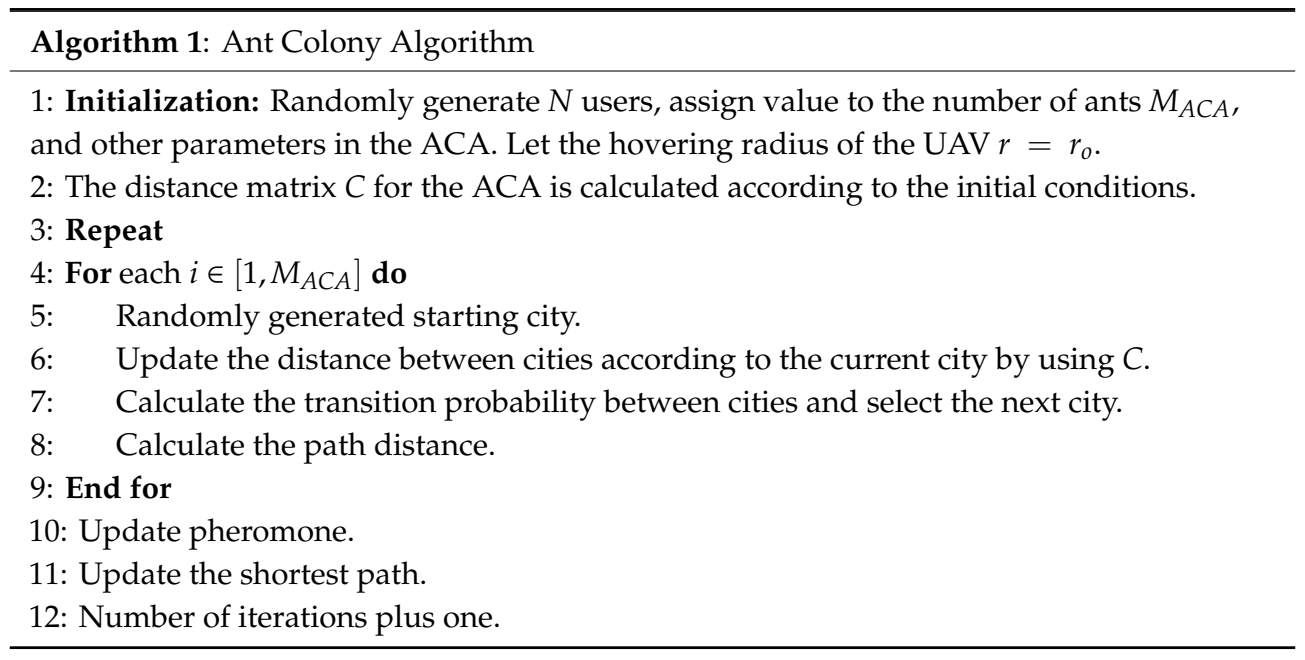

Firstly, a matrix $B_{N \times N}$ is constructed according to the number of users $N$. Each element in the matrix represents the value of the corresponding user objective function within the corresponding time slot, and the element in the matrix $B_{N \times N}$ can be written as follow:

$$
b_{i, j}=\left(S N R_{\max }-S N R_{j}[i]\right)^{2},
$$

where the elements of each row in $B_{N \times N}$ represents the value of the objective function of all users in a certain time slot, and the element of each column represents the value of the objective function of a user in all the time slots.

Secondly, the distance matrix $C$ is calculated for the ACA based on $B_{N \times N}$, and the size of the matrix $C$ is $N^{2} \times N$. The element in the matrix $C$ can be expressed as follows:

$$
c_{i, j}=\left\{\begin{array}{c}
\frac{b_{i-\bmod (i, N)}, \bmod (i, N)}{N}(\bmod (i, N) \neq j) \\
0 \quad(\bmod (i, N)=j)
\end{array} .\right.
$$

Thus, rows $1+N(n-1)$ through the row $N n$ in the matrix $C$ represent the distance between all the cities within the nth time slot.

The core of the algorithm is the iteration to find the optimal path. The specific steps are as follows: Firstly, to build the solution space of the problem, each ant visits each city one by one until it traverses all the cities. Next, the length of each ant's path is calculated. Then, the optimal path in the current iteration is recorded, and the pheromones on the connection paths between the cities are updated in real-time. Finally, after several iterations, the optimal path is found. The distance between cities is constantly updated according to the distance matrix $C$ in the process of the ants visiting cities.

For a given hovering radius $r$ of the UAV, an optimal selection path $D$ can be calculated by this algorithm, where $D$ corresponds to user scheduling, that is, the optimal scheduling of the user under the hovering radius can be obtained by this algorithm. 


\subsection{UAV Hovering Radius Optimization}

Genetic algorithms have many advantages compared with the traditional optimization algorithm, among which the most significant is the capacity to deal with complex problems and parallelism. The genetic algorithm starts from the randomly generated initial solution, and generates new solutions by selecting, crossover, and mutation. Then, the fitness of the new solution is calculated by the fitness function designed, and the next generation population is obtained by natural selection. After several iterations, the algorithm converges to the optimal population.

The genetic algorithm takes the objective function value as the search information directly. It only uses the fitness function value transformed by the objective function value to determine the further search direction and search scope, and does not need some other auxiliary information, such as the derivative value of the objective function.

Genetic algorithms simultaneously use search information from multiple search points. The search for the optimal solution by genetic algorithm starts from an initial group composed of many individuals, rather than a single individual. The fitness function is not only the criterion to measure the merits of individuals, but also the only basis for natural selection. Therefore, the design of the fitness function is particularly important. The genetic algorithm basically does not use external information in evolutionary search, only based on fitness function. The objective function is not constrained by continuous differentiability, and its domain can be any set. The only requirement for the fitness function is that the results can be calculated from the input and compared. The algorithm process is shown in Algorithm 2.

The goal here is to find the optimal radius $r$ for a given user scheduling $A$. The fitness function is designed first. It is assumed that an optimal selection path denoted by $D$ is obtained through the calculation of the ACA. It should be noted that $D$ is a sequence of numbers, which represents the time slot that each user accesses and the size of $D$ is $1 \times N$. The value of the elements in $D$ are 1 to $\mathrm{N}$ and are not repeated. For any given $r$, the corresponding matrix $B_{N \times N}$ can be acquired. Therefore, the fitness function can be expressed as follows:

$$
f(r)=\sum_{i=1}^{N} b_{i, d_{(1, i)}},
$$

where $b$ is an element of matrix $B_{N \times N}$, and $d(1, i)$ is the first row and the $i$-th column element of $D$.

The GA is also called the classical genetic algorithm. Its optimization variables are described by binary coding, and the binary codes of several optimization variables are strung together to form chromosomes. The binary string representing the individual is randomly generated with a certain length of subscript when the initial population is created. The selection operation selects individuals from the old population with a certain probability to form a new population in order to reproduce the next generation of individuals. The probability of an individual being selected is related to its fitness value. The fitness with bigger value will be selected with a higher probability. The crossover operation randomly selects the crossover position on the two chromosomes according to the probability, and exchanges the binary values corresponding to these positions to generate two new individuals. The mutation operation randomly selects the mutation position on the randomly selected individual according to the mutation probability, takes the binary value of the bit, and generates a new individual.

An initial population is first created where the number of individuals is $M_{G A}$. Values are assigned to the crossover probability $P_{c r}$ and the mutation probability $P_{m u}$. The optimal solution in each generation is denoted by OPT. In addition, let gen be the maximum number of iterations.

The parameters $V_{m}$ are set to zero at the beginning of the algorithm. Firstly, the fitness value of each individual in the population is calculated according fitness function. Each population then uses the respective crossover probability $P_{c r}$ and mutation probability $P_{m u}$ generates the next generation through selection, crossover, and mutation. Finally, the individual with the optimal fitness values is found. 


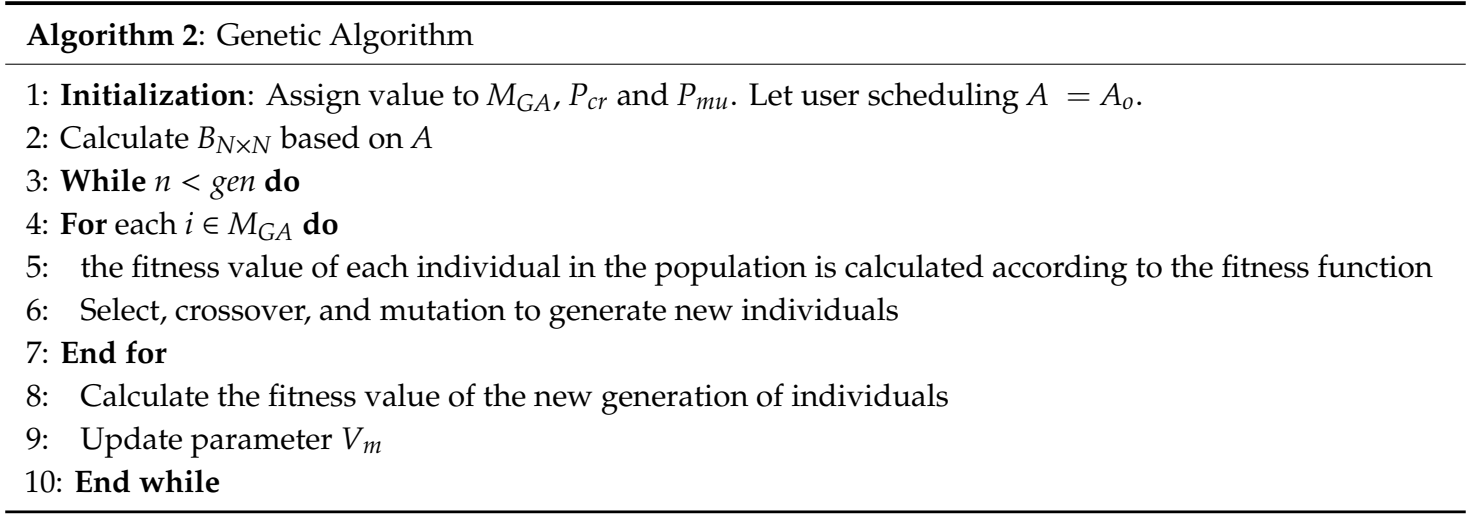

\section{Simulation Results}

This section presents the simulation results to compare the performances of the ACA and the GA. We consider a circular urban area with parameters $\alpha=9.61, \beta=0.16, \eta_{\text {LoS }}=1, \eta_{\text {NLOS }}=20$. The radius of the circular area is $R_{o}$ and the initial value of hovering radius is $r_{0}$. The carrier frequency is $f=20 \mathrm{GHz}$. In addition, it is assumed that the path loss threshold is $L_{t h}=100 \mathrm{~dB}$. The optimal height of the UAV can be calculated according to the maximum path loss. Then, the UAV is placed at this altitude and the user scheduling, along with the hovering radius of the UAV, are further optimized. We first use ACA to optimize user scheduling and then GA to optimize the hovering radius of the UAV. The optimal hovering radius of UAVs maximized the fairness was obtained through iterative optimization of ACA and GA.

\subsection{Convergence Behavior}

An initial value was first assigned to the hovering radius $r_{0}$, and then the optimal user scheduling under the current radius were obtained through the ACA. Then, user scheduling is substituted into the GA to obtain the optimal hovering radius of the UAV under the current user scheduling. In the following simulation figures, the objective function of the vertical axis is the fairness metric we defined in the previous section and smaller value means a better fair performance.

Figure 2 shows the iterative process of the optimal solution when optimizing user scheduling with the ACA. Furthermore, the impact of the different initial radius on the ACA optimization process is shown in the figure. As shown, the initial radius with bigger value will be the smaller value of the objective function optimized by the ACA. This is because the optimal radius was obtained at a certain value in the radius of the target area. The objective function takes the minimum value at the optimal value. Therefore, the initial value with a large difference from the optimal value corresponds to a larger objective function value. Figure 3 shows the iterative process of the optimal solution when optimizing the hovering radius of the UAV with the GA. By comparing Figures 2 and 3, it can be seen that the number of iterations required by the GA to optimize hovering radius of the UAV was less than what was required by the ACA to optimize user scheduling. The problem of optimizing the hovering radius of the UAV is much more complicated than that of optimizing user scheduling, and, therefore, it requires a large number of iterations. 


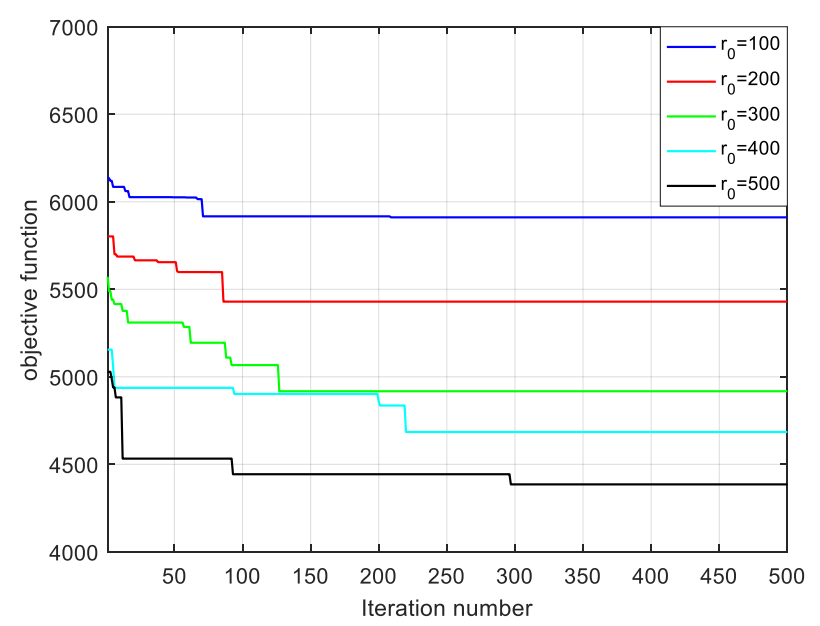

Figure 2. The Iterative process of the Ant Colony Algorithm (ACA).

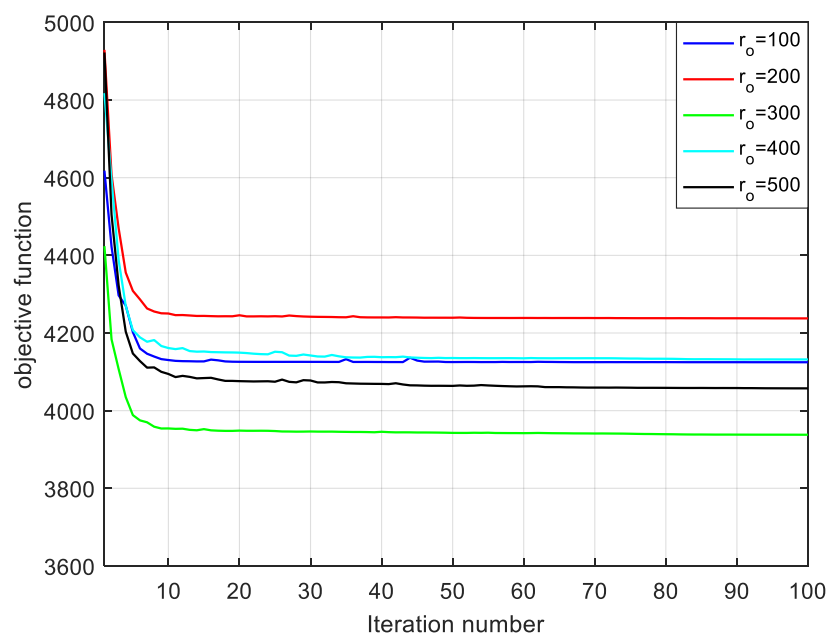

Figure 3. The Iterative process of the Genetic Algorithm (GA).

\subsection{Capacity Performance}

The joint optimization of the hovering radius with a different number of users in the target area is shown in Figure 4 The radius of the target region in Figure $4 \mathrm{a}, \mathrm{b}$ is $1500 \mathrm{~m}$ and $2000 \mathrm{~m}$ respectively. As the number of iterations increases, the hovering radius of UAV converges gradually. As shown, the optimal solution of the algorithm generally does not change after the fourth and fifth iterations, which also shows the effectiveness and stability of the algorithm. In addition, a different number of users $N$ in the area leads to different results of each iteration. The algorithm eventually converges to a value, which is the optimal hovering radius of the UAV in this scenario.

The change process of fairness performance with the number of iterations is shown in Figure 5. The vertical axis of the figure is the fairness metric, which is $\sum_{i=1}^{N} \sum_{n=1}^{N} u_{i}[n]\left(S N R_{\max }-S N R_{i}[n]\right)^{2}$, as defined above; and the fairness metric is the objective function in ACA-GA. The radius of the target region in Figure 5a,b is $1500 \mathrm{~m}$ and $2000 \mathrm{~m}$, respectively. Although the number of users does not affect the convergence of the hovering radius, it does affect the convergence of fairness performance. The fairness performance decreases as the number of user increase. It becomes more difficult to assign each user the time slot that maximizes fairness performance as the number of users increases. 


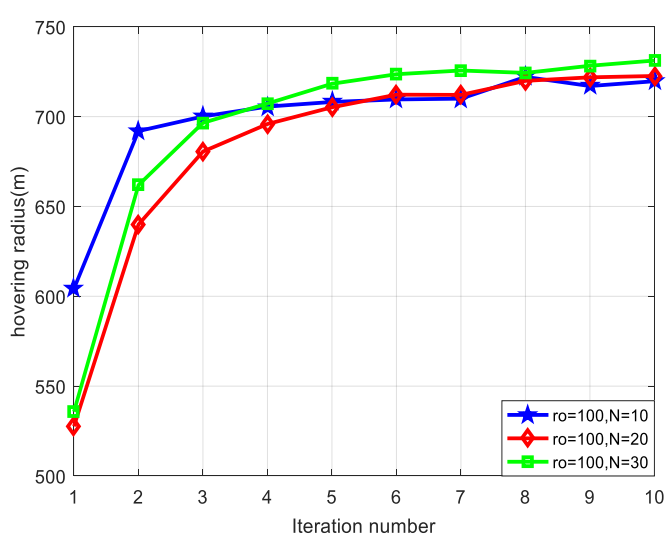

(a)

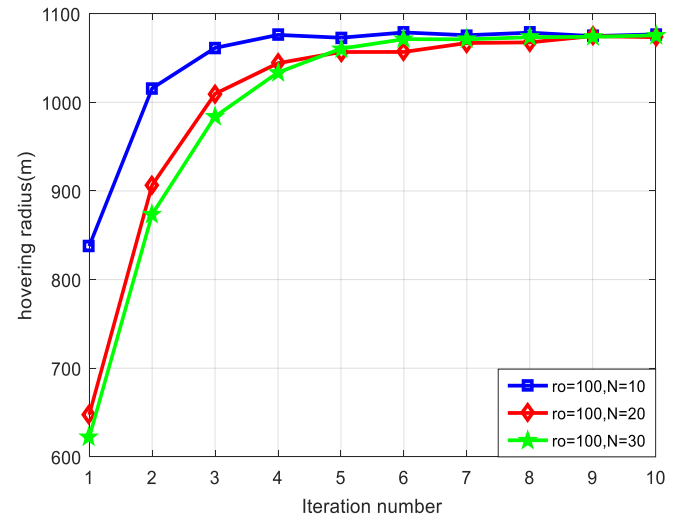

(b)

Figure 4. The iterative process of hovering radius in joint optimization. $R_{o}$ is $1500 \mathrm{~m}$ (a) and $2000 \mathrm{~m}(\mathbf{b})$.

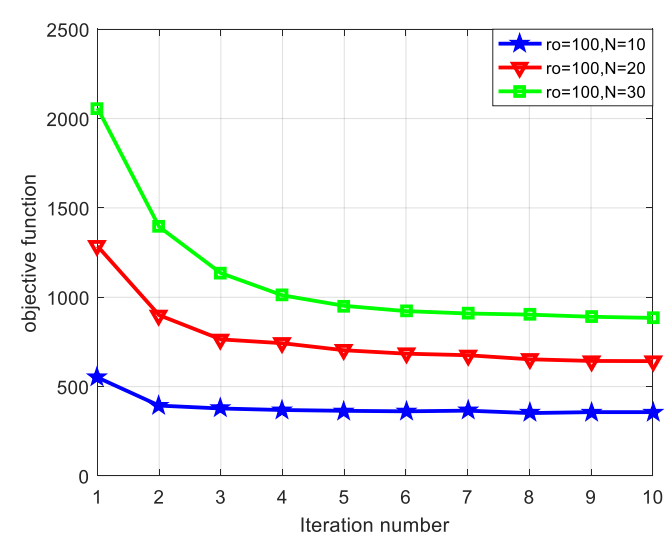

(a)

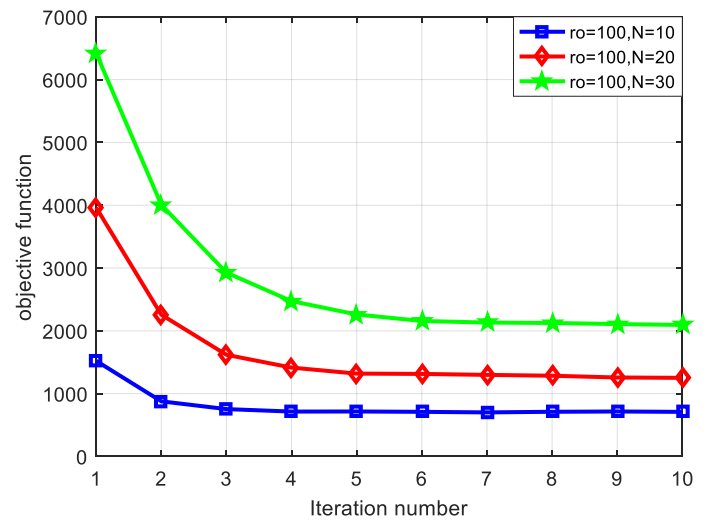

(b)

Figure 5. The iterative process of fairness performance in joint optimization. $R_{o}$ is $1500 \mathrm{~m}$ (a) and $2000 \mathrm{~m}(\mathbf{b})$.

The result, shown in Figure 6, is the network capacity in three different optimization methods. The horizontal line in the figure represents the performance of the system when the UAV is fixed at the regional origin. In this case, no optimization is required, and the total network capacity can be calculated directly from the location of each user, and the result is a fixed value. We can see that when the only genetic algorithm is used to optimize the hovering radius of the UAV without optimizing user scheduling, the performance of the network is lower than that in the case that the UAV is fixed at a point. The reason is that the user is randomly connected to the network during the hovering of the UAV without optimizing user scheduling, and there is no guarantee that each user will have access within the appropriate time slot.

Then we investigate the variation of the throughput with the iteration number in three different optimization methods. We calculate the sum of the transmission rates $C_{\text {sum }}$ based on the SNR of the user $S N R_{i}[n]$ and user scheduling, to represent the throughput of the network. Therefore, $C_{\text {sum }}$ can be written as $C_{\text {sum }}=\sum_{i=1}^{N} \sum_{n=1}^{N} u_{i}[n] \cdot W \log \left(1+S N R_{i}[n]\right) . W$ is the bandwidth and we assume $W=1 \mathrm{MHz}$. As shown in Figure 7, the ACA-GA joint optimization has a significant improvement in the throughput of the network, compared with the case that without optimization and only use GA to optimize radius. ACA-GA converges when the iteration number is 10, while GA converges when the iteration number is about 60. The ACA-GA joint optimization approach has better network capacity and better convergence than either of the above two methods. 


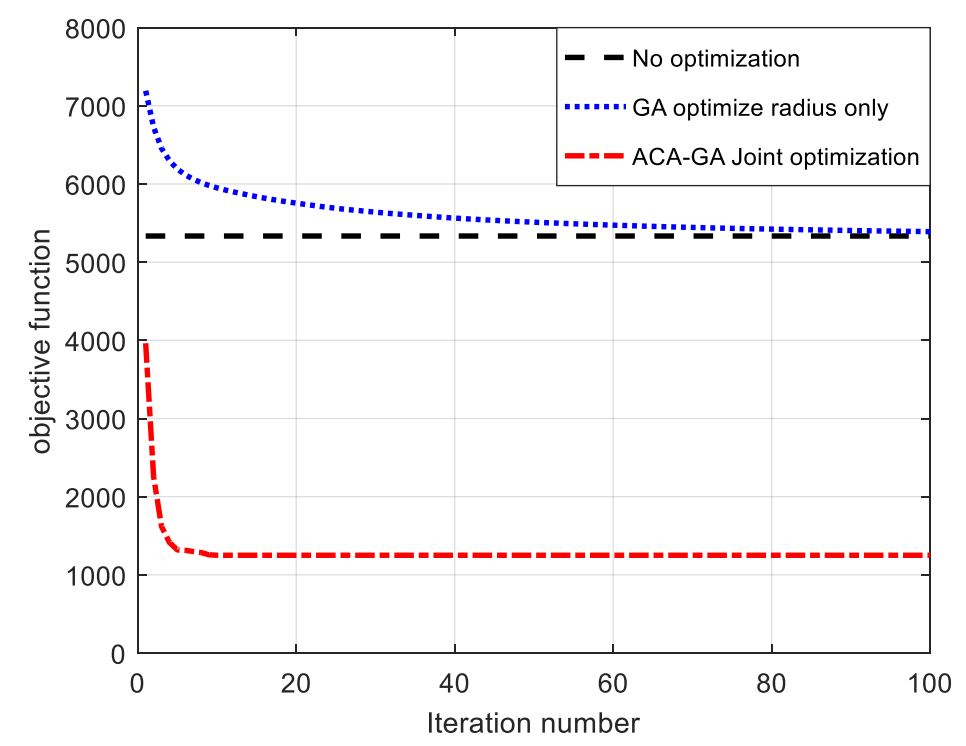

Figure 6. Network capacity in three different optimization methods $(N=20)$.

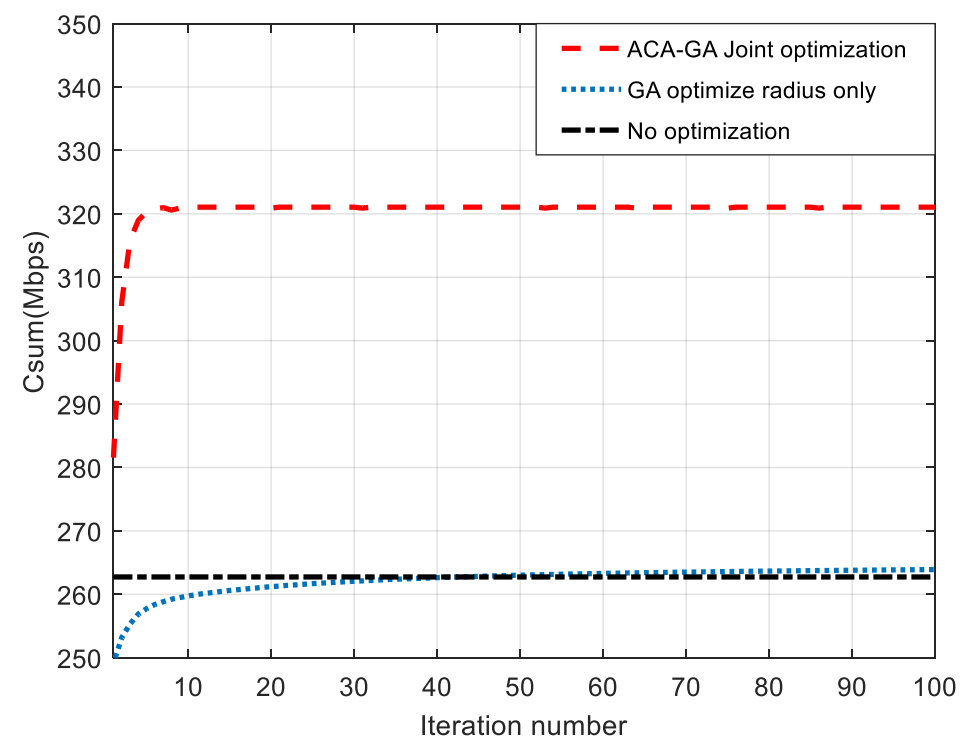

Figure 7. Throughput $C_{\text {sum }}$ in three different optimization methods $(N=20)$.

In order to compare with the ground mobile base station, we consider the situation that the ground mobile base station provides service for users when it moves with a circular trajectory. We adopt the Ground-to-Ground(G2G) channel model, and the channel gain is: $h=\left(\frac{c}{4 \pi f_{c}}\right)^{2} \cdot d^{-k}$, where $c$ is the light speed, $f_{c}$ is the carrier frequency, $d$ is the distance between user and base station, and $k$ is a constant determined by link. The throughput of network is studied at different values of $k$. Figure 8 compares the influence of ground base station and UAV base station on network throughput when $k$ is 3.5 and 4. As shown in the figure, when the ground mobile base station is used to provide services for users, the network throughput is lower than that of the UAV base station. 


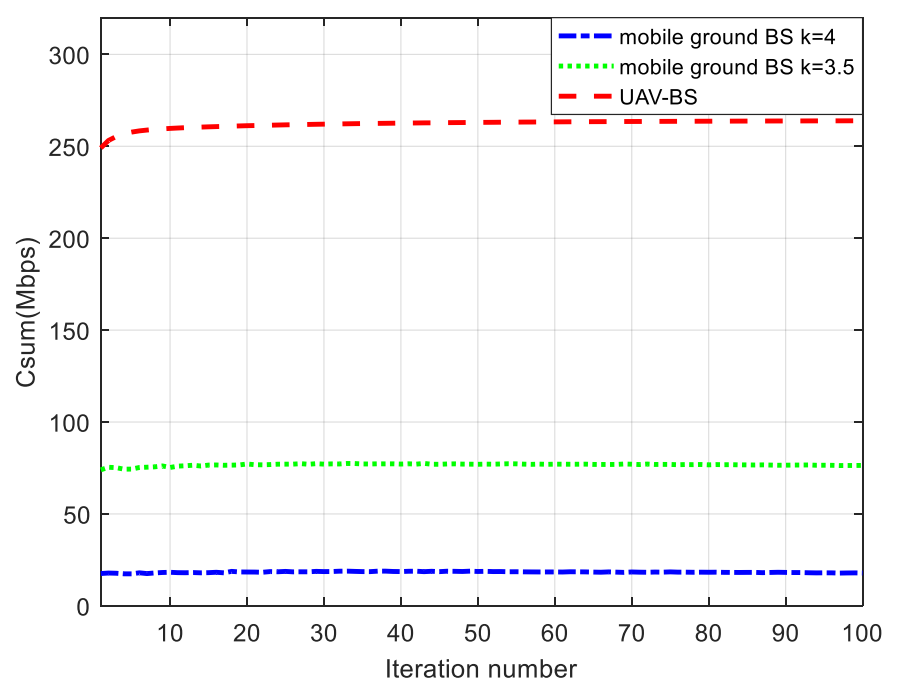

Figure 8. Throughput $C_{\text {sum }}$ of ground base station and UAV-BS.

\section{Conclusions}

In this paper, we investigated the effect of the mobile UAV circular trajectory on network capacity with the consideration of fairness. We considered a UAV-BS hovering with a circular trajectory so that all users in the area can be served. In order to maximize network capacity with the consideration of fairness, trajectory and scheduling of the mobile UAV aerial base station are jointly optimized. We divide the time slots by the number of users, and we take the square sum of the average signal-to-noise ratio of each user within a time slot and the maximal signal-to-noise ratio available to the user as the index to measure fairness. By means of the ACA and the GA, a novel approach of the hovering radius of UAV is proposed, which jointly optimized user scheduling and the hovering radius of the UAV. Simulation results showed that the algorithm proposed in this paper reveals a fast convergence rate and a good convergence result.

Author Contributions: Conceptualization, Y.C. and X.Z.; Data curation, Y.C. and X.Z.; Formal analysis, Y.C. and X.Z.; Funding acquisition, N.L.; Investigation, X.Z.; Methodology, Y.C.; Project administration, N.L.; Resources, N.L. and W.X.; Software, N.L.; Supervision, W.X.; Validation, W.X.; Visualization, W.X.; Writing一original draft, Y.C.; Writing-review and editing, N.L. and X.Z.

Funding: This research was funded by National Natural Science Foundation of China under grant 61871400 and 61571463, the Natural Science Foundation of Jiangsu Province under grant BK20171401 and National Natural Science Foundation of China under grant 61771486.

Conflicts of Interest: The authors declare no conflict of interest.

\section{References}

1. Zeng, Y.; Zhang, R.; Lim, T.J. Wireless communications with unmanned aerial vehicles: Opportunities and challenges. IEEE Commun. Mag. 2016, 54, 36-42. [CrossRef]

2. Bor-Yaliniz, I.; Yanikomeroglu, H. The new frontier in RAN heterogeneity: Multi-tier drone-cells. IEEE Commun. Mag. 2016, 54, 48-55. [CrossRef]

3. Gupta, L.; Jain, R.; Vaszkun, G. Survey of important issues in UAV communication networks. IEEE Commun. Surv. Tuts. 2016, 18, 123-1152. [CrossRef]

4. Mozaffari, M.; Saad, W.; Bennis, M.; Debbah, M. Unmanned aerial vehicle with underlaid device-to-device communications: Performance and tradeoffs. IEEE Trans. Wirel. Commun. 2016, 15, 3949-3963. [CrossRef]

5. Azari, M.; Rosas, F.; Chen, K.C.; Pollin, S. Optimal UAV positioning for terrestrial-aerial communication in presence of fading. In Proceedings of the 2016 IEEE Global Communications Conference (GLOBECOM), Washington, DC, USA, 4-8 December 2016; pp. 1-7.

6. Azari, M.M.; Murillo, Y.; Amin, O.; Rosas, F.; Alouini, M.S.; Pollin, S. Coverage maximization for a poisson field of drone cells. IEEE PIMRC 2017, arXiv:1708.06598v1. 
7. Guo, Z.; Wei, Z.; Feng, Z.; Fan, N. Coverage probability of multiple UAVs supported ground network. Electron. Lett. 2017, 53, 885-887. [CrossRef]

8. Mozaffari, M.; Saad, W.; Bennis, M.; Debbah, M. Mobile Internet of Things: Can UAVs provide an energy-efficient mobile architecture? In Proceedings of the IEEE Global Communications Conference GLOBECOM, Washington, DC, USA, 4-8 December 2016; pp. 1-6.

9. Mozaffari, M.; Saad, W.; Bennis, M.; Debbah, M. Drone small cells in the clouds: Design, deployment and performance analysis. In Proceedings of the 2015 IEEE Global Communication Conference (GLOBECOM), San Diego, CA, USA, 6-10 December 2015; pp. 1-6.

10. Mozaffari, M.; Saad, W.; Bennis, M.; Debbah, M.E. Optimal Transport Theory for Power-Efficient Deployment of Unmanned Aerial Vehicles. In Proceedings of the IEEE International Conference on Communications (ICC), Kuala Lumpur, Malaysia, 22-27 May 2016; pp. 22-27.

11. Zhan, P.; Yu, K.; Swindlehurst, A.L. Wireless relay communications with unmanned aerial vehicles: Performance and optimization. IEEE Trans. Aerosp. Electron. Syst. 2011, 47, 2068-2085. [CrossRef]

12. Zeng, Y.; Zhang, R.; Lim, T.J. Throughput maximization for UAV-enabled mobile relaying systems. IEEE Trans. Commun. 2016, 64, 4983-4996. [CrossRef]

13. Akarsu, A.; Girici, T. Fairness aware multiple drone base station deployment. Commun. IET 2018, 12, 425-431. [CrossRef]

14. Al-Hourani, A.; Kandeepan, S.; Lardner, S. Optimal LAP altitude for maximum coverage. IEEE Wirel. Commun. Lett. 2014, 3, 569-572. [CrossRef]

15. Bor-Yaliniz, I.; El-Keyi, A.; Yanikomeroglu, H. Efficient 3-D placement of an aerial base station in next generation cellular networks. In Proceedings of the IEEE International Conference Communication (ICC), Kuala Lumpur, Malaysia, 22-27 May 2016; pp. 1-5.

16. Zhang, X.; Duan, L. Optimization of emergency UAV deployment for providing wireless coverage. In Proceedings of the GLOBECOM 2017-2017 IEEE Global Communications Conference, Singapore, Singapore, 4-8 December 2017; pp. 1-6.

17. Alzenad, M.; El-keyi, A.; Lagum, F.; Yanikomeroglu, H. 3D placement of an unmanned aerial vehicle base station (UAV-BS) for energy-efficient maximal coverage. IEEE Wirel. Commun. Lett. 2017, 6, 1-4. [CrossRef]

18. Alzenad, M.; El-Keyi, A.; Yanikomeroglu, H. 3D Placement of an Unmanned Aerial Vehicle Base Station for Maximum Coverage of Users with Different QoS Requirements. IEEE Wirel. Commu. Lett. 2018, 7, 38-41. [CrossRef]

19. Wang, F.; Chen, W.; Tang, H.; Wu, Q. Oint optimization of user association, subchannel allocation, and power allocation in multi-cell multi-association OFDMA heterogeneous networks. IEEE Trans. Commun. 2017, 65, 2672-2684. [CrossRef]

20. Zeng, Y.; Zhang, R. Energy-efficient UAV communication with trajectory optimization. IEEE Trans. Wirel. Commun. 2017, 16, 3747-3760. [CrossRef]

21. Lyu, J.; Zeng, Y.; Zhang, R. Cyclical multiple access in UAV-aided communications: A throughput-delay tradeoff. IEEE Wirel. Commun. Lett. 2016, 5, 600-603. [CrossRef]

22. Nesterov, Y.E.; Todd, M.J. Self-scaled barriers and interior-point methods for convex programming. Math. Oper. Res. 1997, 22, 1-4. [CrossRef]

(C) 2019 by the authors. Licensee MDPI, Basel, Switzerland. This article is an open access article distributed under the terms and conditions of the Creative Commons Attribution (CC BY) license (http://creativecommons.org/licenses/by/4.0/). 